\title{
Handbook of Anaesthesia and Peri-Operative Medicine
}

\author{
Cyprian Mendonca, Chandrashekhar Vaidyanath (Editors). tfm Publishing Ltd., 2017, \\ £45.00, paperback, 840 pages. ISBN: 978-1-910079-19-5
}

\author{
Kaitlin Duncan, MD $\mathbb{D}$
}

Received: 30 August 2017/Accepted: 3 October 2017/Published online: 10 October 2017

(C) Canadian Anesthesiologists' Society 2017

As anesthesiology continues to expand its reach beyond the borders of the operating room, increasing responsibility will fall on the upcoming generations of anesthesiologists to develop a skill set able to support the scope of this broadened practice. It is of paramount importance, therefore, that medical students and junior residents gain an earlier and broader understanding of anesthesia in the perioperative setting.

Although the Handbook of Anaesthesia \& Perioperative Medicine may be their first foray into this topic, Dr. Cyprian Mendonca and co-author Dr. Chandrashekhar Vaidyanath clearly have a wealth of experience writing and compiling textbooks for junior learners. At just over 800 pages, the term "handbook" may be overstating its portability. The large text size, colourcoded section headings, and real-life photographs, however, make this textbook a visually engaging read. This book - intended as a general reference for senior medical students and junior residents - aims to promote a holistic understanding of the intersection between anesthesiology, surgery, and perioperative medicine. As the book was written in the United Kingdom, North American learners should take note that many of the references, although not significantly different, refer to European guidelines.

The Handbook of Anaesthesia \& Peri-operative Medicine is structured into seven sections: Applied Science, Peri-Operative Medicine, Airway Management, Specialty-Specific Procedures, Peri-Operative Emergencies, Critical Care Medicine, and Pain Medicine.

K. Duncan, MD (ه)

University of Ottawa, Ottawa, ON, Canada

e-mail: kait.duncan@gmail.com
Each section contains multiple chapters that address individual topics pertinent to anesthesiology and perioperative medicine. The table of contents is well organized to reflect this division. One of the highlights of this book is the inclusion of case scenarios at the end of each chapter. The scenarios are often comprised of one simple case and a second, more-complex case, each serving to illustrate a particular concept in a clinical setting. This case-based approach not only provides an interactive and educational style for the target audience, it serves as a valuable teaching resource for staff and senior residents.

The authors were tasked with covering a broad range of topics, with the consequence that certain sections are necessarily brief. Throughout the textbook, however, there is continued emphasis on clinical relevance. This is especially evident in the Applied Science section, particularly in the excellent chapter on perioperative monitoring. This chapter reviews not only the use of basic anesthetic monitors but also commonly encountered problems associated with them and how they could affect one's clinical interpretation. The Peri-operative Medicine section holds its own as the title's namesake, boasting (arguably) that it is the best chapter in the book on perioperative fluid therapy. This chapter's clinical approach to "debunking" the myths of fluid therapy, its practical, concise overview of recent literature, and the applicability of its case scenarios should make this chapter a go-to for any junior (and many senior) anesthesiology residents. The Airway Management section is a singularly long chapter and, owing to its breadth of topics, may be more challenging to navigate. It suffers somewhat from a lack of conciseness and could benefit from better use of tables and figures to demonstrate concepts. The chapter, however, is written conversationally 
and at an appropriate level to convey a basic understanding of airway management. The Specialty-Specific Procedures section broadly covers all major subspecialties and includes an interesting chapter on considerations for emergency surgical procedures. A good resource for any Resident, the Peri-operative Emergencies section provides useful clinical algorithms. The section on Critical Care Medicine is equally pertinent and references recent research and guidelines. The section on Pain Medicine nicely reviews the physiology and management of chronic pain but does not mention acute pain. Apart from brief mention in case scenarios on analgesic drugs and local anesthetics, acute pain management as a perioperative focus is largely omitted. This important topic may warrant further discussion in future editions.

When compared with other textbooks for a similar audience, such as the Ottawa Anesthesia Primer ${ }^{\mathrm{A}}$ (previously: Anesthesia for Medical Students), Mendonca and Vaidyanath's Handbook performs very well. What it may lack in detailed discussion of basic anesthesia principles, it more than makes up for in its comprehensive review of perioperative management and subspecialty anesthesia. Although longer than a true pocketbook, such as the NMS Clinical Manual of Anesthesiology, ${ }^{\mathrm{B}}$ it remains comfortably more compact than the Oxford Handbook of Anaesthesia ${ }^{\mathrm{C}}$ - a reference book with similar breadth of content.

Overall, the authors are to be commended for compiling a book that is true to its title as it succeeds in highlighting the complexities of preoperative, intraoperative, and postoperative care in an anesthetic context. The wellreferenced content and practical case scenarios are effectively targeted to a medical student and junior resident level, although the style and organization of key concepts make it a worthwhile refresher text for senior trainees as well. Overall, the Handbook of Anaesthesia \& Peri-operative Medicine is a stimulating addition to the anesthesia trainee's arsenal and will undoubtedly help grow their interest in the field of perioperative medicine.

Conflicts of interest None declared.

Editorial responsibility This submission was handled by Dr. Hilary P. Grocott, Editor-in-Chief, Canadian Journal of Anesthesia. $\overline{\text { A Sullivan P }}$. Ottawa Anesthesia Primer. Toronto: Echo Book Publishing; 2012.
$\overline{\mathrm{B} \text { Glidden } R S}$. NMS Clinical Manual of Anesthesiology. Baltimore: Wolters Kluwer; 2002.

C Allman KG, Wilson IH, O’Donnell A. Oxford Handbook of Anaesthesia. Oxford: Oxford University Press; 2016. 